\title{
Wound rotor induction generator bearing fault modelling and detection using stator current analysis
}

\author{
Damian S. Vilchis-Rodriguez, Sinisa Djurović, Alexander C. Smith \\ School of Electrical and Electronic Engineering, The University of Manchester, Power Conversion Group, \\ Sackville Street Building, Manchester M13 MPL, UK \\ E-mail: Damian.Vilchis-rodriguez@manchester.ac.uk
}

\begin{abstract}
This study investigates wound rotor induction machine bearing fault detection by stator current analysis. The research first establishes an analytic machine model that enables high fidelity simulation of a range of machine bearing defects. The timestepped simulation results are then used to examine stator current spectral signatures of typical bearing faults. The calculations indicate that a number of low magnitude fault specific frequency components appear in the current signal as a result of air-gap variations produced by an incipient bearing fault. However, the considerably low magnitude levels at which these components are exhibited would make the detection of bearing fault using conventional current signature analysis techniques challenging. An alternative technique based on spectral analysis of complex current signals is therefore proposed in order to improve fault detection. The validity of the findings of this work is confirmed by analysis of measured data obtained on a $30 \mathrm{~kW}$ commercial machine test rig that can be configured to introduce a range of different bearing fault severities.
\end{abstract}

\section{Introduction}

A large proportion of modern wind power generation cost is related to wind turbine (WT) operation and maintenance, with unscheduled maintenance cost reported to account for as much as $75 \%$ of the overall maintenance expense [1]. In order to make the wind energy systems economically feasible in comparison with conventional means of power generation it is clearly necessary to minimise unscheduled downtime and associated reparation costs, and especially so in hostile off-shore environments. One of the means of achieving this is through developing effective condition-based maintenance techniques that allow the operator to obtain knowledge of incipient system failures and plan for required maintenance in advance.

Traditional fault surveys in induction machines (IMs) indicate that close to $40 \%$ of all IM failures are linked to bearing failures [2]. Incidentally, a large proportion of reported WT failures are also associated with bearing faults in the generator and gearbox [1]. Recent surveys on modern WT failures also highlight the generator shaft bearing fault as one of the most significant in the WT drive train $[1,3]$. For example, reported operational statistical data for wind generators above $2 \mathrm{MW}$ indicate that $58 \%$ of total generator failures originate from bearing faults [3]. Wound rotor induction machines (WRIMs) remain one of the most frequently used machine types for electromechanical energy conversion in modern large variable speed WT drives and thus achieving early detection of faults in these machines is of considerable importance. Research on the development of non-invasive techniques for detection of electrical and mechanical faults in WRIMs, therefore has recently started receiving increased attention [4-10]. A shaft bearing fault disturbs the electro-mechanical symmetry in the generator, resulting in dynamic and/or static changes to the generator air-gap compared to healthy operating conditions. The mechanical nature of a bearing fault is such that it yields predictable frequencies in the machine vibration spectrum that can be related to the bearing geometry and fault location [10-12]. Bearing fault detection methods have, therefore traditionally favoured vibration analysis techniques [10] and most commercially available WT generator condition monitoring systems rely solely on vibration signal analysis [13]. However, this approach comes with a penalty of requiring specialised invasive sensors for vibration signal monitoring and data acquisition. Alternatively, shaft bearing faults will also induce changes in the machine symmetry by introducing minute periodic air-gap variations that, due to the coupled nature of the machine electromagnetic system, affect the machine electrical output signals. The generator currents, for example, can be expected to contain embedded information concerning the nature and the extent of the bearing fault and, therefore have the potential to be used either instead of or complementary to the vibration signals for bearing fault detection [7-12]. The potential advantage of using electrical signal analysis stems from the fact that these are readily available within the WT control system and, therefore require no additional sensors and ensuing infrastructure for monitoring.

Bearing fault analysis in cage rotor IMs using electrical signals has received significant attention, however, there are very few papers focusing on WRIMs [7-9]. The lack of sensitivity of stator current analysis for bearing fault detection however has been recognised as the limiting 
factor in the successful application of traditional current signature analysis (CSA) methods in the detection of bearing faults. Therefore sophisticated post-processing techniques have been applied to the current signal in DFIGs in order to improve fault detection [7-9]. For instance, in [7] empirical mode decomposition is applied to the stator current and further processing of the first intrinsic mode function allow the construction of a statistical based index for enhanced fault detection of localised bearing faults. The approach in [7] is then validated experimentally using a small test machine in a pollution free electric environment. In [8], wavelet filtering is used for the detection of generalised roughness in the machine bearings using stator current and an index is built for enhanced bearing fault detection. This paper investigates, the possibility of achieving the drive end shaft bearing fault detection in a commercial WRIM by spectral analysis of stator current-based signals. The proposed fault signals have the potential to be further enhanced using more sophisticated post-processing techniques as the used in $[7,8]$.

The research develops a dynamic WRIM analytical model based on principles of generalised harmonic analysis and complex conductor distribution $[14,15]$ that accounts for air-gap field disturbances introduced by the presence of a typical outer race bearing fault. In order to improve current signal spectral signature-based fault detection, this work proposes a monitoring technique that employs spectral analysis of complex current signals. A laboratory test-rig using a commercial $30 \mathrm{~kW}$ WRIM that can be reconfigured to emulate a range of bearing faults is used for experimental validation of the derived model and to demonstrate the effectiveness of the proposed approach.

\section{Bearing fault modelling}

Deep grove ball bearings are commonly used in wind generators because of their tolerance to axial load. The geometry analysed in this work corresponds to this specific bearing type, however, the modelling approach may be adopted to suit alternative rolling bearing types.

\subsection{Bearing frequencies}

Depending on the number of rolling elements contained in a particular bearing design, the geometric relationship between its components and their rotational speed, a set of characteristic rotational frequencies $\left(f_{\mathrm{c}}\right)$ that originate from respective bearing components can be defined. In this research, the characteristic frequencies of interest are the inner $\left(f_{\mathrm{i}}\right)$ and outer race $\left(f_{\mathrm{o}}\right)$ passing frequencies which are given by (1) and (2), in $[10,11]$

$$
\begin{aligned}
& f_{\mathrm{i}}=f_{\mathrm{r}} \frac{N_{b}}{2}\left(1+\frac{D_{b}}{D_{c}} \cos \varphi\right) \\
& f_{\mathrm{o}}=f_{\mathrm{r}} \frac{N_{b}}{2}\left(1-\frac{D_{b}}{D_{c}} \cos \varphi\right)
\end{aligned}
$$

where $N_{b}$ is the number of rolling elements, $D_{b}$ is the rolling element diameter, $D_{c}$ is the bearing pitch diameter, $\varphi$ is the contact angle and $f_{\mathrm{r}}$ is the shaft rotational speed. The existing bearing fault location (inner or outer race) will determine which of the characteristic frequencies described by (1) and (2) will be exhibited in the machine vibration signals. In vibration analysis, therefore the passing frequency becomes the main fault frequency. This bearing asymmetry will also result in a modulation of the machine air-gap thus disturbing the symmetry of the field distribution and consequently modulating the machine electrical signals.

\subsection{Machine model}

In order to gain an in-depth understanding of how bearing fault effects are manifested in the investigated commercial machine design and examine the potential of applying stator CSA for detection of WRIM incipient bearing faults, a detailed machine coupled-circuit model was established. The model is based on the principles presented in [14-16] and extends these to take into account the machine bearing ball passing frequency as well as the width and depth of the investigated bearing defect. It should be noted that in general the ball passing frequency is a non-integer quantity yielding faulty machine time-varying inductances that are non-periodic between consecutive shaft rotations. The derivation of analytical expressions that enable reliable estimation of faulty machine coupling is therefore of utmost relevance for credible representation of fault effects.

In deriving the model it is assumed that an incipient bearing fault starts as a localised fault that is therefore simulated in the model by considering temporary air-gap eccentricity variations, with the eccentricity appearing each time a rolling element passes over the defective area in the bearing. This is then reflected in the model calculations as a periodic air-gap variation related to the ball passing frequency. The model derivation considers an outer race fault, which may be modelled as a form of periodic static eccentricity. To account for an inner race fault periodic dynamic eccentricity must be assumed in the modelling stage instead [11]. The presented work focuses on investigation of WRIMs and the modelling approach would need to be modified in order to accommodate similar analysis of other machine types.

For the considered bearing outer race fault, the air-gap variation may be expressed in terms of the static eccentricity [11] by assuming a time-variant eccentricity function $\left(\delta_{\mathrm{se}}\right)$ that is rotor displacement dependant

$$
g(y, t)=g_{0}-g_{0} \delta_{\mathrm{se}}(\beta(t)) \cos (y)
$$

where $g_{0}$ is the mean air-gap, $\beta$ is the linear displacement between the stator and the rotor reference frames and $y$ is the location around the machine mean air-gap periphery.

The extent of the fault induced air-gap variation in the machine depends on the geometric relationship between the defective surface width $\left(w_{\mathrm{d}}\right)$ and depth $\left(b_{\mathrm{d}}\right)$ and the ball bearing radius $\left(r_{\mathrm{b}}\right)$, as illustrated in Fig. 1 . In the analysis it is assumed that the depth of the fault $\left(b_{\mathrm{d}}\right)$ is sufficient to

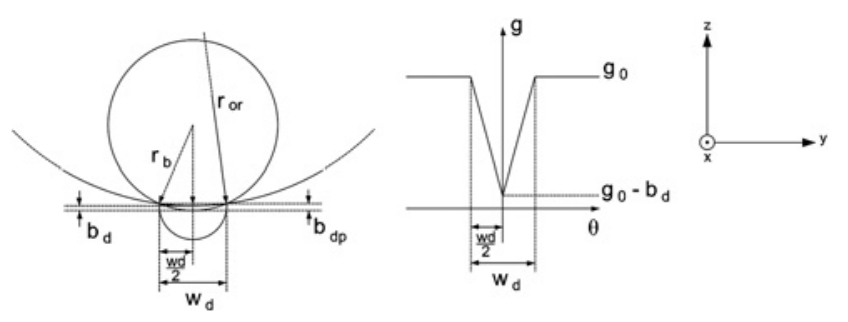

Fig. 1 Air-gap variation 
allow the radial displacement of the rolling element to be limited by the defect width only. Other geometric and physical constraints such as bearing clearance, race shape and cage rigidity are not considered in the model. The model, therefore assumes an effective shaft displacement based on geometrical considerations only. It should also be noted that the model neglects any mechanical reaction between the ball and the defective area.

In addition, a bearing fault will result in periodic air-gap variations at the faulty end of the machine only and will therefore also give rise to a varying axial asymmetry along the air-gap. The effects of this on the machine can be characterised by considering a uniform axial eccentricity equal to the average of the eccentricity at the ends of the machine [17]. If it is assumed that the non-faulted end remains centred, for the incipient bearing fault case considered here, the resulting shaft displacement may be assumed to be small and thus extremely small eccentricity variations would be expected. The inverse of the air-gap function for the incipient fault scenario can thus be conveniently approximated with the following expression

$$
g^{-1}(y, t) \simeq \frac{1}{g_{0}}\left[1+\delta_{\mathrm{se}}(\beta(t)) \cos (y)\right]
$$

2.2.1 Inductance calculation: Considering an $m$ th stator winding excited with an arbitrary time varying current $i_{m}(t)$ and applying the principles of Ampere's circuit law over the integration path $L$ in Fig. 2, the resulting air-gap magnetic flux density $B_{z}$ to surface current density $J_{x}$ relationship may be expressed as

$$
\frac{\partial\left[B_{z}(y, t) g(y, t)\right]}{\partial y}=\mu_{0} J_{x}
$$

where $\mu_{0}$ is the permeability of the free space and $g(y, t)$ is a general expression that accounts for an arbitrary fault induced air-gap variation. The surface current density $J_{x}$ may now be written in terms of the $m$ th winding complex conductor distribution [7] and the current it carries

$$
\frac{\partial\left[B_{z}(y, t) g(y, t)\right]}{\partial y}=\mu_{0} i_{m}(t) \sum_{v=-\infty}^{\infty} \overline{C_{\mathrm{s} m}^{k}} \mathrm{e}^{-\mathrm{j} k y}
$$

where $\overline{C_{\mathrm{s} m}^{k}}$ is the $v$ th harmonic complex conductor distribution of the $m$ th stator circuit and the wave number, $k=2 v / d$, where $d$ is the mean air-gap diameter [14]. From (4) and (6) the magnetic flux density in the air-gap produced by current $i_{m}(t)$ is obtained as

$$
\begin{aligned}
B_{z}(y, t)= & \frac{\mu_{0} i_{m}(t)}{g_{0}}\left[\sum_{v=-\infty}^{\infty} \mathrm{j} \frac{\overline{C_{\mathrm{s} m}^{k}}}{k} \mathrm{e}^{-\mathrm{j} k y}+\frac{1}{2} \delta_{\mathrm{se}}(t) \sum_{v=-\infty}^{\infty} \mathrm{j} \frac{\overline{C_{\mathrm{s} m}^{k}}}{k} \mathrm{e}^{-\mathrm{j}(k-1) y}\right. \\
& \left.+\frac{1}{2} \delta_{\mathrm{se}}(t) \sum_{v=-\infty}^{\infty} \mathrm{j} \frac{\overline{C_{\mathrm{s} m}^{k}}}{k} \mathrm{e}^{-\mathrm{j}(k+1) y}\right]
\end{aligned}
$$

The electric field produced on the stator surface by current $i_{m}(t)$ flowing through the $m$ th stator winding, therefore is
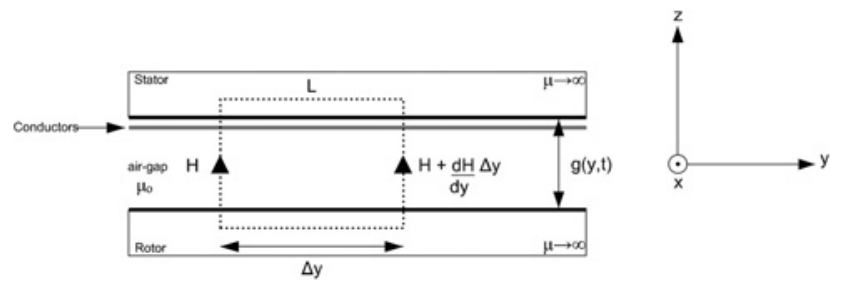

Fig. 2 Linearised machine geometry

$$
\begin{aligned}
E_{x}= & \frac{\mathrm{d}}{\mathrm{d} t}\left[\frac { \mu _ { 0 } i _ { m } ( t ) } { g _ { 0 } } \left(\sum_{v=-\infty}^{\infty} \frac{\overline{C_{\mathrm{s} m}^{k}}}{k^{2}} \mathrm{e}^{-\mathrm{j} k y}+\frac{1}{2} \delta_{\mathrm{se}}(t) \sum_{v=-\infty}^{\infty} \frac{\overline{C_{\mathrm{s} m}^{k}}}{k(k-1)} \mathrm{e}^{-\mathrm{j}(k-1) y}\right.\right. \\
& \left.\left.+\frac{1}{2} \delta_{\mathrm{se}}(t) \sum_{v=-\infty}^{\infty} \frac{\overline{C_{\mathrm{s} m}^{k}}}{k(k+1)} \mathrm{e}^{-\mathrm{j}(k+1) y}\right)\right]
\end{aligned}
$$

The EMF induced in a patch $\Delta y$ of an arbitrary stator $n$th winding with conductors distribution $C_{\mathrm{s} n}(y)$ originating from the electric field produced by the $m$ th stator winding excitation can now be obtained from

$$
U(t)=\int_{0}^{\pi d} w E_{x}(y) C_{\mathrm{s} n}(y) \mathrm{d} y
$$

producing

$$
\begin{aligned}
U(t)= & \frac{\mathrm{d}}{\mathrm{d} t}\left[\frac { \mu _ { 0 } w \pi d i _ { m } ( t ) } { g _ { 0 } } \left(\sum_{v=-\infty}^{\infty} \frac{\overline{C_{\mathrm{s} m}^{k}} \frac{*}{C_{\mathrm{s} n}^{k}}}{k^{2}}\right.\right. \\
& +\frac{1}{2} \delta_{\mathrm{se}}(t) \sum_{v=-\infty}^{\infty} \frac{\overline{C_{\mathrm{s} m}^{k}} \frac{*}{C_{\mathrm{s} n}^{k-1}}}{k(k-1)} \\
& \left.\left.+\frac{1}{2} \delta_{\mathrm{se}}(t) \sum_{v=-\infty}^{\infty} \frac{\overline{C_{\mathrm{s} m}^{k}}}{k(k+1)}\right)\right]
\end{aligned}
$$

The mutual inductance between any two arbitrary stator windings $m$ and $n$ can, therefore be defined as

$L_{\mathrm{s} m, \mathrm{~s} n}(t)=\frac{\mu_{0} w \pi d}{g_{0}}\left[\sum_{v=-\infty}^{\infty} \frac{\overline{C_{\mathrm{s} m}^{k}} \frac{*}{C_{\mathrm{s} n}^{k}}}{k^{2}}+\frac{1}{2} \delta_{\mathrm{se}}(t) \sum_{\nu=-\infty}^{\infty} \frac{\overline{C_{\mathrm{s} m}^{k}} \frac{*}{C_{\mathrm{s} n}^{k \pm 1}}}{k(k \pm 1)}\right]$

Using the same approach it can be shown that the inductance between any two arbitrary rotor windings $m$ th and $n$th is defined by

$$
\begin{aligned}
L_{\mathrm{r} m, \mathrm{r} n}(t)= & \frac{\mu_{0} w \pi d}{g_{0}}\left[\sum_{\nu=-\infty}^{\infty} \frac{\overline{C_{\mathrm{r} m}^{k}} \frac{*}{C_{\mathrm{r} n}^{k}}}{k^{2}}\right. \\
& \left.+\frac{1}{2} \delta_{\mathrm{se}}(t) \sum_{\nu=-\infty}^{\infty} \frac{\overline{C_{\mathrm{r} m}^{k}} \frac{*}{C_{\mathrm{r} n}^{k+1}}}{k^{\mp \mathrm{j} \beta(t)}} \mathrm{e}^{\mp 1)}\right]
\end{aligned}
$$

whereas the inductance between an arbitrary stator $m$ th 
winding and an arbitrary rotor $n$th winding is given by

$$
\begin{aligned}
L_{\mathrm{s} m, \mathrm{r} n}(t)= & \frac{\mu_{0} w \pi d}{g_{0}}\left[\sum_{v=-\infty}^{\infty} \frac{\overline{C_{\mathrm{s} m}^{k}} \frac{*}{C_{\mathrm{r} n}^{k}} \mathrm{e}^{-\mathrm{j} k \beta(t)}}{k^{2}}\right. \\
& +\frac{1}{2} \delta_{\mathrm{se}}(t) \sum_{v=-\infty}^{\infty} \frac{\overline{C_{\mathrm{s} m}^{k}} \frac{*}{C_{\mathrm{r} n}^{k \pm 1}}}{\left.\mathrm{e}^{-\mathrm{j}(k \pm 1) \beta(t)}\right]}
\end{aligned}
$$

In contrast to inductance expressions defined in a similar manner for the healthy WRIM operation [15], the expressions in (11)-(13) indicate that, for operation under a bearing fault, all the machine inductances become time-varying. The nature of these inductance variations is determined by the assumed eccentricity variation function $\delta_{\text {se }}$ used to replicate investigated bearing fault conditions. The derived harmonic inductance expressions can now be used to formulate a time-stepping machine mathematical model similar to that in [15] that enables detailed analysis of the fault spectral signature on the machine electrical quantities.

2.2.2 Eccentricity function for bearing outer race fault: The eccentricity variation function $\delta_{\text {se }}$ is periodic as the fault induced air-gap variations occur each time that a rolling element passes over the bearing race defective area. The period of $\delta_{\mathrm{se}}$ is given by $T=\pi d / f_{\mathrm{f}}$, where $f_{\mathrm{f}}$ is the number of times that a rolling element passes over the defective area per mechanical revolution. For the bearing outer race defects investigated in this work $f_{\mathrm{f}}$ is obtained by dividing the characteristic fault frequency defined by (1) and (2) and the rotational frequency $f_{\mathrm{r}}$. A period of the assumed eccentricity variation function is shown in Fig. 3 for the machine geometry considered in Fig. 1. In terms of complex Fourier series, the eccentricity variation may be expressed as

$$
\delta_{\mathrm{se}}(t)=\sum_{\ell=-\infty}^{\infty} C_{\mathrm{se}}^{\ell} \mathrm{e}^{\mathrm{j} \eta f_{\mathrm{f}} \beta(t)}
$$

where $\ell$ is the harmonic order and

$$
\eta=\frac{2 \ell}{d}
$$

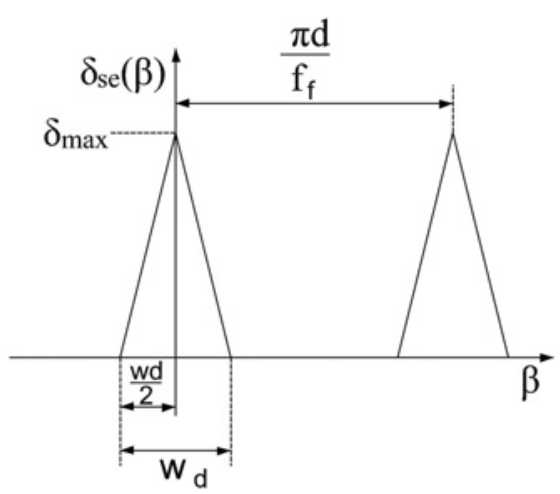

Fig. 3 Eccentricity variation function for $\ell=0$

$$
C_{\mathrm{se}}^{0}=\frac{\delta_{\mathrm{max}} w_{\mathrm{d}} f_{\mathrm{f}}}{2}
$$

and for any other $\ell$

$$
C_{\mathrm{se}}^{\ell}=\frac{\delta_{\text {max }} d}{\pi \ell^{2} w_{\mathrm{d}} f_{\mathrm{f}}}\left[1-\cos \left(\frac{\ell w_{\mathrm{d}} f_{\mathrm{f}}}{d}\right)\right]
$$

where $\delta_{\max }$ is the maximum eccentricity. The presented analysis was developed for an outer race fault, however, the described approach can also accommodate an inner race fault by considering a dynamic eccentricity air-gap function with a time variant eccentricity function instead of that assumed in (3).

\subsection{Complex signal envelope frequency spectrum}

The existence of a localised bearing fault will yield additional components in the stator current spectrum at frequencies $\mid f_{\mathrm{s}} \pm$ $k_{n} f_{\mathrm{c}} \mid$ [10], where $f_{\mathrm{s}}$ is the fundamental supply frequency and $k_{n}$ is the harmonic order of the characteristic frequency $f_{\mathrm{c}}$, which is defined by (1) and (2) for an inner or outer race fault, respectively. Under such conditions, the modulated stator current can be expressed in the time domain as

$$
\begin{aligned}
i_{\mathrm{s}}(t)= & i_{m} \cos \left(\omega_{\mathrm{s}} t+\alpha\right)+i_{f d} \cos \left(\omega_{\mathrm{s}} t-\omega_{\mathrm{c}} t+\xi\right) \\
& +i_{f u} \cos \left(\omega_{\mathrm{s}} t+\omega_{\mathrm{c}} t+\gamma\right)
\end{aligned}
$$

where $i_{m}$ is the magnitude of the fundamental, $i_{f d}$ and $i_{f u}$ are the magnitude of the lower and upper sideband components of the modulated signal and $\omega_{\mathrm{s}}=2 \pi f_{\mathrm{s}}$ and $\omega_{\mathrm{c}}=2 \pi f_{\mathrm{c}}$ are the respective system and fault related angular frequencies, with arbitrary phase angles given by $\alpha, \xi$ and $\gamma$. If the current signal in (18) is delayed by an arbitrary time period $\Delta t$

$$
\begin{aligned}
i_{\mathrm{s}}(t-\Delta t)= & i_{m} \cos \left(\omega_{\mathrm{s}}(t-\Delta t)+\alpha\right) \\
& +i_{f d} \cos \left(\omega_{\mathrm{s}}(t-\Delta t)-\omega_{\mathrm{c}}(t-\Delta t)+\xi\right) \\
& +i_{f u} \cos \left(\omega_{\mathrm{s}}(t-\Delta t)+\omega_{\mathrm{c}}(t-\Delta t)+\gamma\right)
\end{aligned}
$$

Choosing $\Delta t$ to be equivalent to a quarter of a cycle of the fundamental supply frequency, the time delayed signal in (19) becomes

$$
\begin{aligned}
i_{\mathrm{s}}(t-\Delta t)= & i_{m} \cos \left(\omega_{\mathrm{s}} t+\alpha-\frac{\pi}{2}\right) \\
& +i_{f d} \cos \left(\omega_{\mathrm{s}} t-\omega_{\mathrm{c}} t+\xi+\frac{\omega_{\mathrm{c}}}{\omega_{\mathrm{s}}} \frac{\pi}{2}-\frac{\pi}{2}\right) \\
& +i_{f u} \cos \left(\omega_{\mathrm{s}} t+\omega_{\mathrm{c}} t+\gamma-\frac{\omega_{\mathrm{c}}}{\omega_{\mathrm{s}}} \frac{\pi}{2}-\frac{\pi}{2}\right)
\end{aligned}
$$

Expanding further produces

$$
\begin{aligned}
i_{\mathrm{s}}(t-\Delta t)= & i_{m} \sin \left(\omega_{\mathrm{s}} t+\alpha\right) \\
& +i_{f d} \sin \left(\omega_{\mathrm{s}} t-\omega_{\mathrm{c}} t+\xi+\frac{\omega_{\mathrm{c}}}{\omega_{\mathrm{s}}} \frac{\pi}{2}\right) \\
& +i_{f u} \sin \left(\omega_{\mathrm{s}} t+\omega_{\mathrm{c}} t+\gamma-\frac{\omega_{\mathrm{c}}}{\omega_{\mathrm{s}}} \frac{\pi}{2}\right)
\end{aligned}
$$


The main purpose of this development is to reduce the interference caused by the dominant supply frequency component $i_{m}$ while preserving the fault signature information. Based on the orthogonal property of sinusoidal signals, this information can be encapsulated in a complex signal defined in terms of (18) and (21). The complex current signal is therefore defined as

$$
I_{\mathrm{s}}(t)=i_{\mathrm{s}}(t)+j i_{\mathrm{s}}(t-\Delta t)
$$

where $j$ is the imaginary operator. After further mathematical manipulation, it can be shown that the square of the modulus of the complex current signal in (22) has the following form

$$
\begin{aligned}
i_{\mathrm{s}}(t)^{2}+i_{\mathrm{s}}(t-\Delta t)^{2}= & i_{m}^{2}+i_{f d}^{2}+i_{f u}^{2}+i_{\omega_{\mathrm{c}}}(t) \\
& +i_{\omega_{\mathrm{c}} \pm 2 \omega_{\mathrm{s}}}(t)+i_{2 \omega_{\mathrm{c}}}(t)+i_{2\left[\omega_{\mathrm{c}} \pm \omega_{\mathrm{s}}\right]}(t)
\end{aligned}
$$

where sub-indices in the time variant quantities of (23) indicate the frequency components present in the corresponding element. The derivation of the elements of (23) is given in Appendix 1. It should be noted that the modulus of the defined complex signal is actually the signal envelope. The expression in (23) demonstrates that the frequency spectrum of the envelope of the complex current signal contains, in terms of harmonic fault frequencies, the fault related components $k_{n} f_{\mathrm{c}}, k_{n} f_{\mathrm{c}} \pm 2 f_{\mathrm{s}}$, $2 k_{n} f_{\mathrm{c}}$ and $2\left(k_{n} f_{\mathrm{c}} \pm f_{\mathrm{s}}\right)$, whereas the DC component is formed mainly by the magnitude of the fundamental supply component, thus the fundamental power supply component is rejected.

The bearing fault specific frequency magnitudes in the current signal are generally of very low magnitude when compared to the current fundamental. The magnitudes of the fault related frequency components in the envelope signal given by $k_{n} f_{\mathrm{c}}$ and $k_{n} f_{\mathrm{c}} \pm 2 f_{\mathrm{s}}$, however, are effectively scaled up by the fundamental magnitude, making these fault indicators more pronounced in the envelope signal spectrum. In addition, the noise inherently present in a measured signal is naturally averaged during the procedure, signal averaging is a common technique used to improve the signal-to-noise ratio (SNR) in weak signals. The presented procedure results therefore in a potential improvement in the detection of the existing low magnitude bearing fault indicators in the machine current. It should be noted that expression (23) is valid for any modulating frequency including those that appear as a result of mixed eccentricity or other inherent unbalance conditions. In general, therefore additional frequencies linked to these other modulating signals will also appear in the envelope spectrum.

\subsection{Instantaneous symmetrical components}

It was found in this work that the bearing fault spectral signature can differ significantly between the three stator line currents in the machine. In order to account for this, the instantaneous negative sequence signal defined in terms of real or complex input signals, may be used as a means of averaging the magnitude of the fault frequency components in all three machine currents. Instantaneous symmetrical components may be defined by

$$
\left[\begin{array}{l}
I_{0}(t) \\
I_{1}(t) \\
I_{2}(t)
\end{array}\right]=\frac{1}{3}\left[\begin{array}{ccc}
1 & 1 & 1 \\
1 & a & a^{2} \\
1 & a^{2} & a
\end{array}\right]\left[\begin{array}{c}
i_{a}(t) \\
i_{b}(t) \\
i_{c}(t)
\end{array}\right]
$$

where $I_{0}, I_{1}$ and $I_{2}$ are the instantaneous zero, positive and negative symmetrical components, respectively, $i_{a}, i_{b}$ and $i_{c}$ are the real valued or complex instantaneous phase signals and $a$ is the symmetrical operator, $a=\mathrm{e}^{\mathrm{j} 2 \pi / 3}$. When real valued signals are used as input in (24) the transformation yields instantaneous symmetrical components as defined in [18]. Alternatively, if a set of complex current signals defined as in (22) is transformed by (24), instantaneous symmetrical components akin to those defined in terms of the analytic complex signals [19] are obtained. In both approaches, the overall effect of the unbalanced operation is accounted for in the resulting negative sequence component. It should be noted that since the appearance of negative sequence is also related to asymmetric operating conditions, a temporary increase in the negative sequence component magnitude is to be expected to take place every time a bearing ball passes over the defective surface. Hence, when a bearing fault is present, a frequency component at the ball passing frequency would be expected to appear in the frequency spectrum of the instantaneous magnitude of the negative sequence component.

\section{Simulation results}

The sensitivity of CSA for bearing fault detection was first investigated using the model developed in Section 2.2. Detailed industrial laboratory machine specifications were used as data inputs for the developed coupled-circuit model [15]. The machine parameters are given in Appendix 2. An arbitrary operating speed of $1600 \mathrm{rpm}$ and a nominal sinusoidal balanced voltage supply were used in the simulated results. An outer race bearing fault was simulated using the model with a characteristic fault frequency of 3.07 times the machine rotational speed and a range of fault severities. The simulated characteristic frequency corresponds to the SKF 6313 roller bearings used in the test machine.

As mentioned in Section 2.2, the non-integer nature of the ball passing frequency expressed by (1) and (2) results in non-periodic inductances. This is illustrated in Fig. 4, where the calculated mutual inductance and its derivative between a stator phase winding and one of the rotor phase branches are shown for an assumed $1 \mathrm{~mm}$ width bearing fault for two consecutive shaft revolutions. No visible fault induced change can be observed in predictions for the inductance distribution; however, the fault effects are pronounced and can be clearly identified in predictions for the inductance spatial derivative. However, when the coupling between a complete stator winding and a rotor phase winding is considered, the overall effect of the fault is seen to be negligible in both the inductance and its derivative, as illustrated in Fig. 5 for a considered $7 \mathrm{~mm}$ fault. The inductance derivatives are proportional to the electromagnetic torque signal and consequently affect the machine currents. The presented data, therefore demonstrate that the fault spectral signature in WRIM line current can be expected to be of low magnitude. In addition, the data suggest that in a machine with multiple parallel branches, 


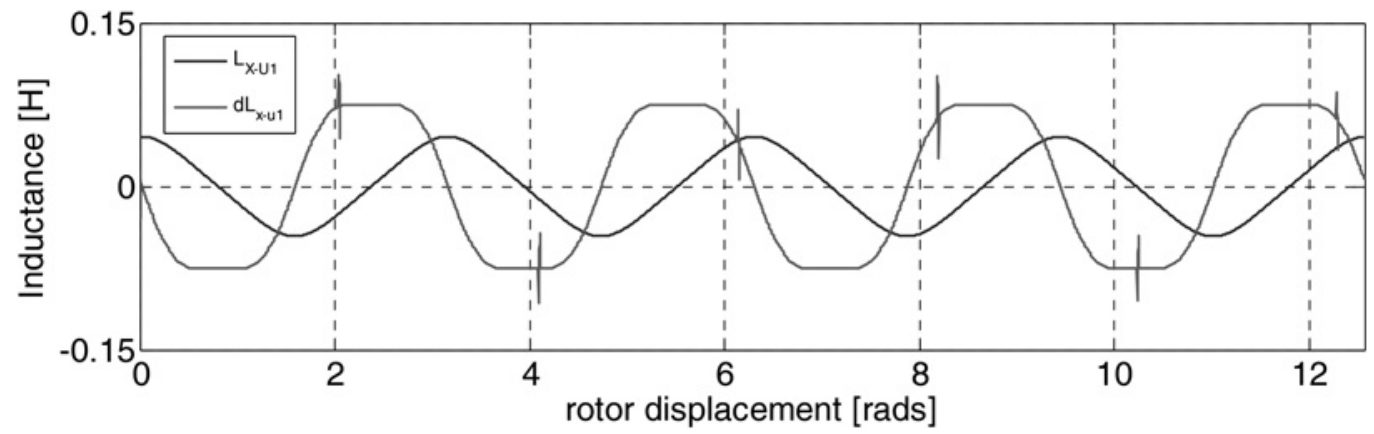

Fig. 4 Stator phase $X$ winding rotor phase branch U1 mutual inductance and its derivative

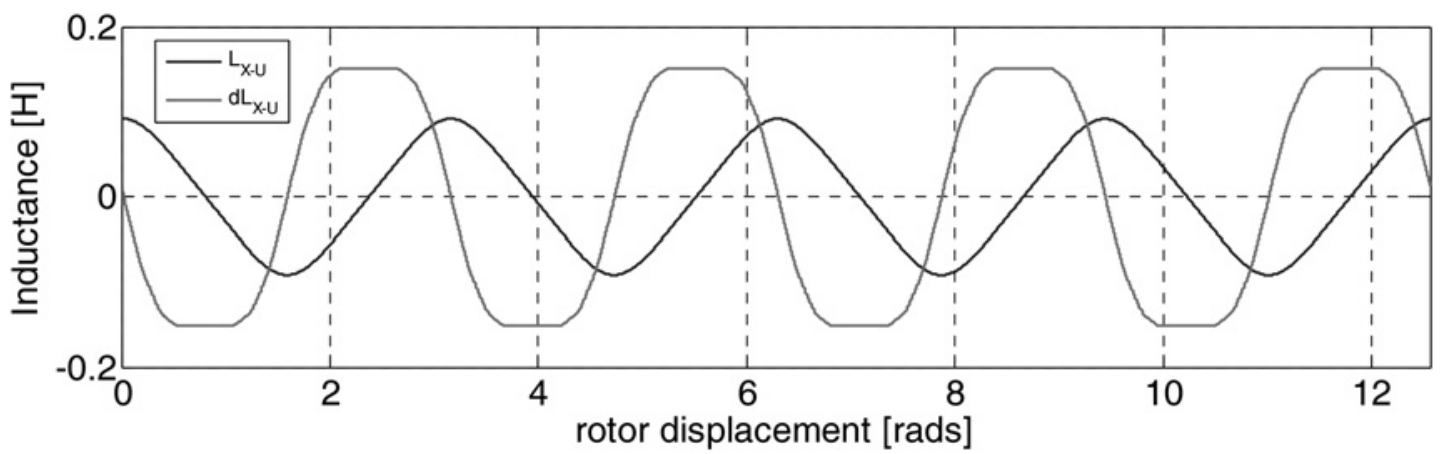

Fig. 5 Stator phase X rotor phase U mutual inductance and its derivative

the branch quantities may be more sensitive to exhibiting the bearing failure induced spectral effects.

Model predictions for healthy and faulty WRIG stator current spectra are shown in Fig. 6 for an assumed mechanical speed of 1600 RPM and different fault severities ranging from 1 to $7 \mathrm{~mm}$, corresponding to fault induced air-gap variation at the drive-end of the machine in the range of $1-75 \%$ of the air-gap length. Fig. 7 shows a detailed view of the principal fault component.

For the outer race bearing fault analysed, $f_{\mathrm{c}}=f_{\mathrm{o}}$ and thus the fault frequencies in the current signal are given by $\left|f_{\mathrm{s}} \pm k_{n} f_{\mathrm{o}}\right|$ (25). The characteristic outer race frequency was calculated to be $f_{\mathrm{o}}=81.86 \mathrm{~Hz}$. The considered low severity bearing faults (1-3 mm) produce small air-gap variations with a peak value of less than $13 \%$ of air-gap length at the faulted end of the machine. The predicted current data in Fig. 7 indicate that fault effects are not significant in the current signal spectrum. The presented model results clearly illustrate that even under simulated balanced and noise free sinusoidal supply conditions, the amplitudes of the bearing fault specific current frequencies are significantly lower in comparison with those of the fundamental and other existing spectral components. Their detection for purposes of fault indication would, therefore not be trivial. It is also

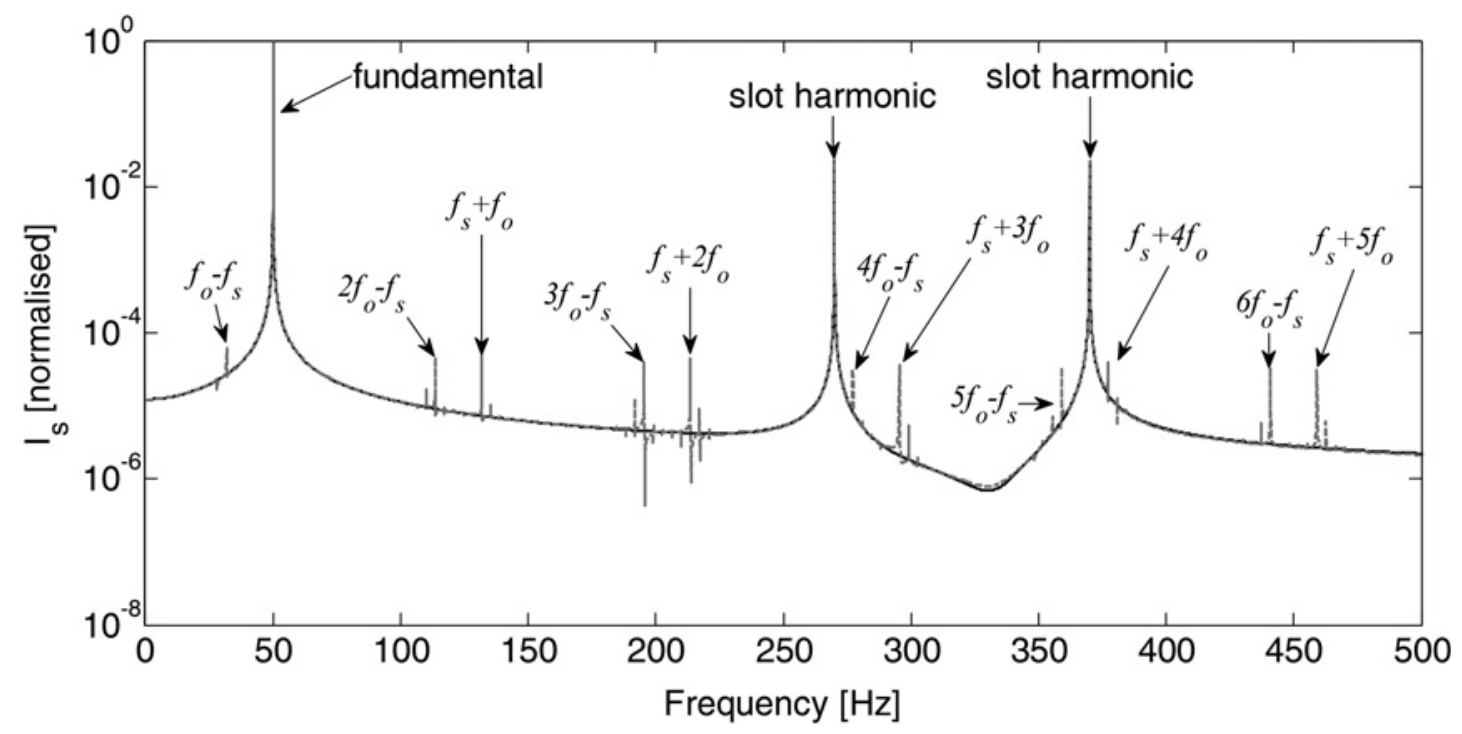

Fig. 6 Stator current frequency spectra for differing fault severity, simulation results $1600 \mathrm{rpm}$ 


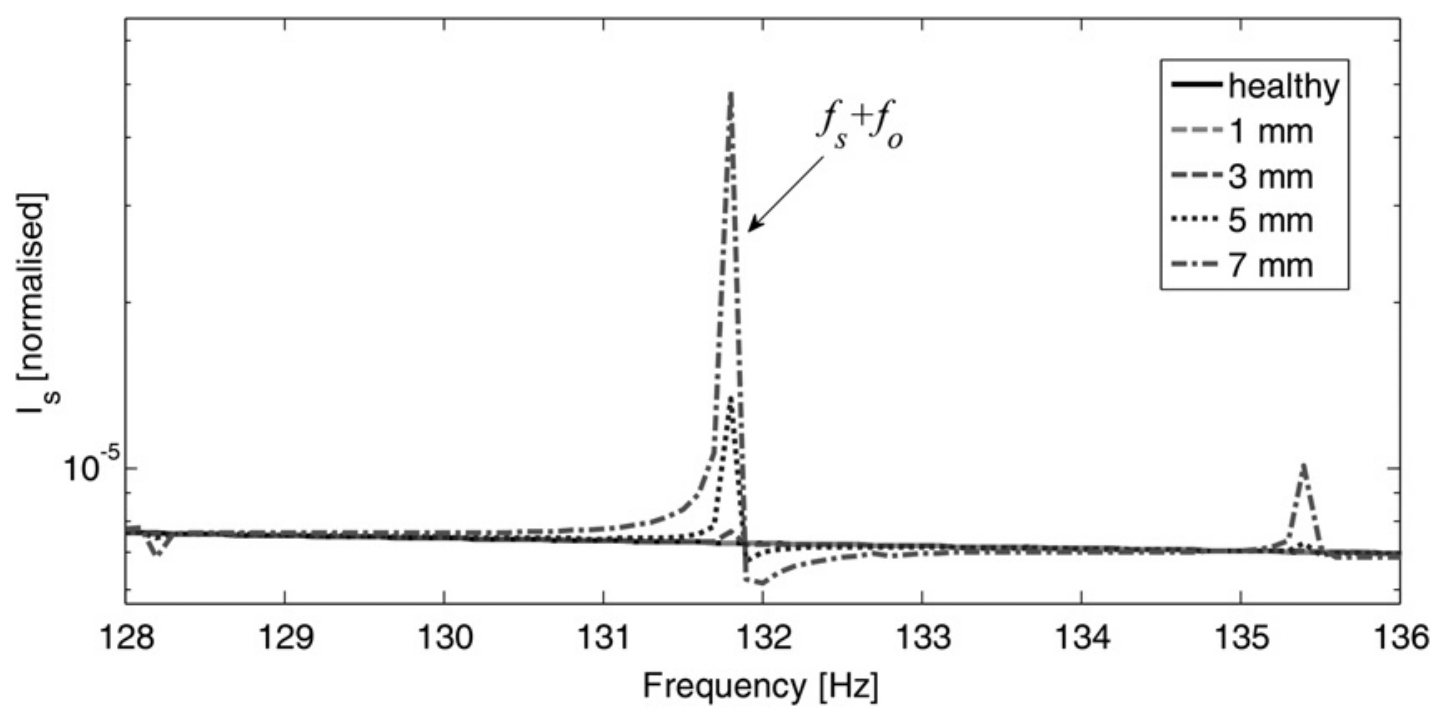

Fig. 7 Stator current frequency spectrum, principal fault component

interesting to observe in the figures that the model predicts the appearance of very low magnitude sideband frequencies around the fundamental and principal fault frequencies resulting from the faulty machine operation.

For simulated fault widths from 3 to $7 \mathrm{~mm}$ corresponding to 23 to $75 \%$ eccentricity at the faulted end of the machine, the simulation results clearly indicate the presence of fault related frequencies in the stator current spectrum. The amplitude of the predicted fault frequencies is seen to be extremely low and is in the order of $0.005 \%$ of the fundamental. The identified fault frequencies are apparent in the simulation results where no noise presence is considered, however, their detection would be significantly and adversely affected by any presence of noise in the current signal.

\section{Experimental results}

The experimental work was based on the results obtained from a test rig comprising a $30 \mathrm{~kW}$ industrial WRIM with short circuited rotor windings driven at constant speed by a DC motor. The machine bearings specifications are given in Table 1 and the machine parameters are shown in Appendix 2. Bearing faults were experimentally simulated by drilling a hole in the middle of the bearing outer race perpendicular to the race surface on the drive-end side bearing, as illustrated in Fig. 8. A Bruel\&Kjær Pulse system was used to record the vibration signal with one accelerometer fitted to the load side end-plate thus providing a comparison between fault effects in mechanical and electrical machine signals.

The stator line current was recorded for a series of experiments at arbitrary steady-state operating speeds ranging from 1530 to $1630 \mathrm{rpm}$. The measured stator

Table 1 Machine bearing data

\begin{tabular}{lc}
\hline Drive-end & Non-drive-end \\
\hline SKF 6313 & SKF 6214 \\
$N_{b}=8$ & $N_{b}=10$ \\
$f_{\mathrm{O}}=3.07 f_{\mathrm{r}}$ & $f_{\mathrm{O}}=4.11 f_{\mathrm{r}}$ \\
$f_{\mathrm{i}}=4.93 f_{\mathrm{r}}$ & $f_{\mathrm{i}}=5.89 f_{\mathrm{r}}$ \\
\hline
\end{tabular}

current frequency spectra are shown in Fig. 9 for healthy machine operation and for machine operation with a $12 \mathrm{~mm}$ hole in the outer race bearing at $1600 \mathrm{rpm}$. It should be noted that compared to the model simulation, the shaft displacement produced using this approach is considerably restricted by the bearing geometry and its physical characteristics. Thus, although the magnitude of the induced fault seems extreme, its effect on the machine air-gap is rather moderate in practice. The locations of the dominant bearing fault specific frequencies, $k_{n}=1$ and $k_{n}=2$ in (25), are indicated in the graph.

Fig. 9 demonstrates that even a severe fault does not produce significant effects in the current signal spectrum. The fault indicators are seen to be comparable in magnitude to the existing noise levels in the recorded spectra and their efficient detection is impaired by the presence of a number of larger spectral components and the low SNR in the bandwidth of interest. On the other hand, as illustrated in Fig. 10, bearing fault frequencies are evident in the corresponding measured vibration spectrum, producing noticeable increments for the $f_{\mathrm{o}}$ and $2 f_{\mathrm{o}}$ components with respect to healthy machine operation. The presented results

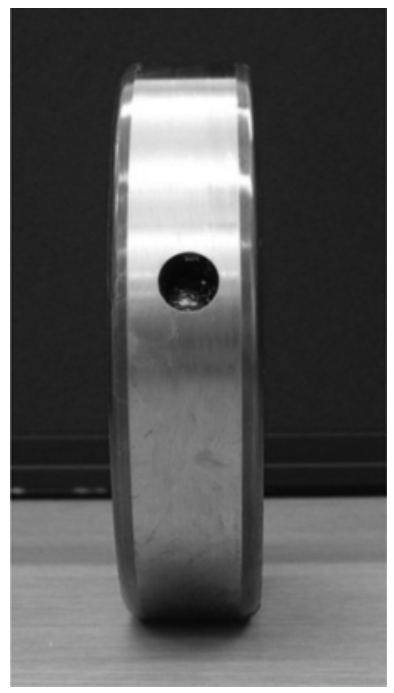

Fig. 8 Faulted bearing 


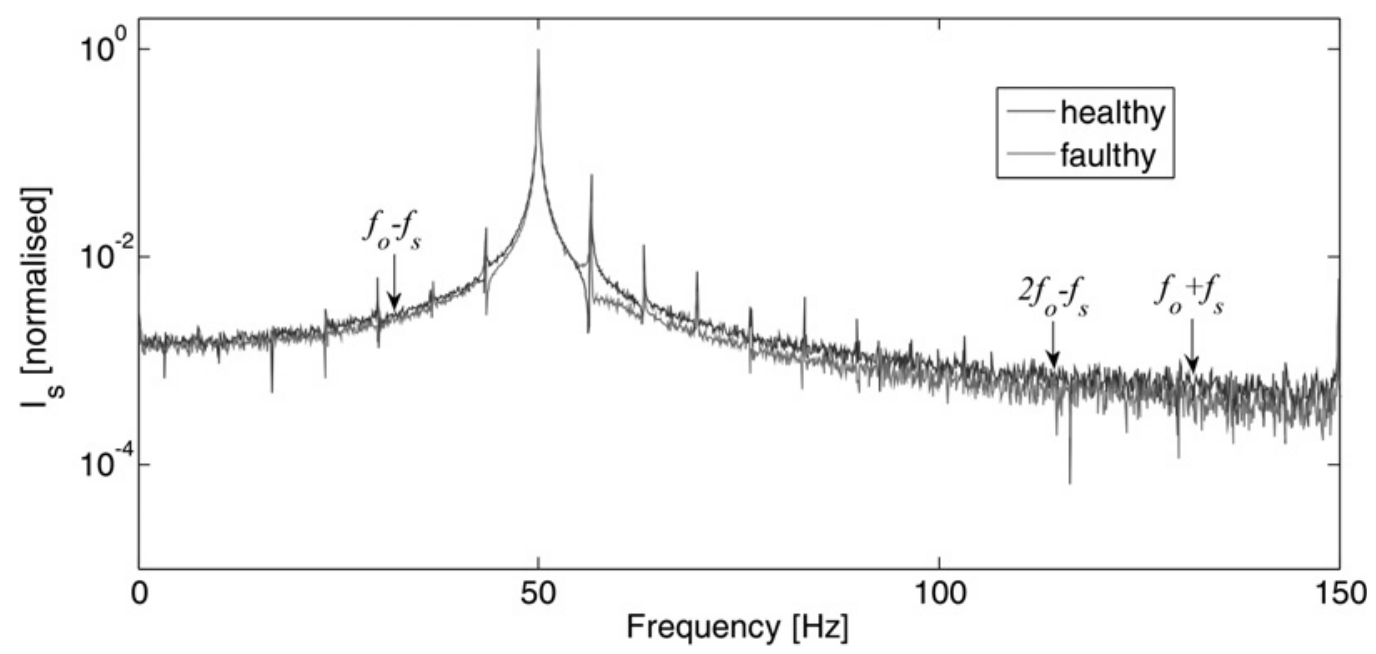

Fig. 9 Stator current frequency spectra at $1600 \mathrm{rpm} 12 \mathrm{~mm}$ hole, experimental results

indicate that the bearing fault modulates the machine air-gap but is largely undetectable using CSA under nominal operating conditions.

It should be noted that although the laboratory machine operating speed in Fig. 10 is $1600 \mathrm{rpm}$, a minor offset exists between the measured fault frequency and its theoretical value. For instance, in accordance with the rotational speed and bearing specification at $1600 \mathrm{rpm}$, the expected fault frequency is $81.86 \mathrm{~Hz}$, whereas the actual fault frequency measured in the vibration spectrum was $82.34 \mathrm{~Hz}$. This offset partly originates from inaccuracies in the speed measurement system and may lead to confusion if additional frequencies exist in the vicinity of the theoretical fault frequency.

A comparison of the measured faulty machine stator current spectrum and its complex current envelope frequency spectrum is given in Fig. 11. The results indicate a clear improvement in the detection of the fault frequency, originating from use of the signal envelope spectrum analysis. The fault specific frequency component is clearly identifiable in the envelope spectrum of the faulty machine signal, thus validating the potential of the approach proposed in Section 2.3. Although the use of the envelope frequency spectrum facilitates the detection of bearing

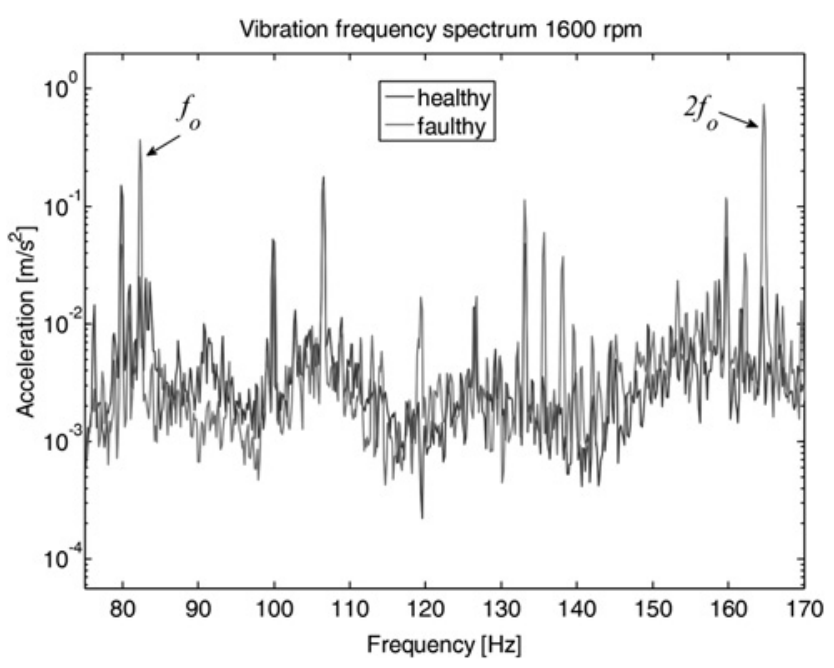

Fig. 10 Vibration frequency spectrum, $1600 \mathrm{rpm} 12 \mathrm{~mm}$ hole faults, this technique relies on the analysis of a single machine current signal, where the presence of a particular type of fault may be masked, as the fault components are
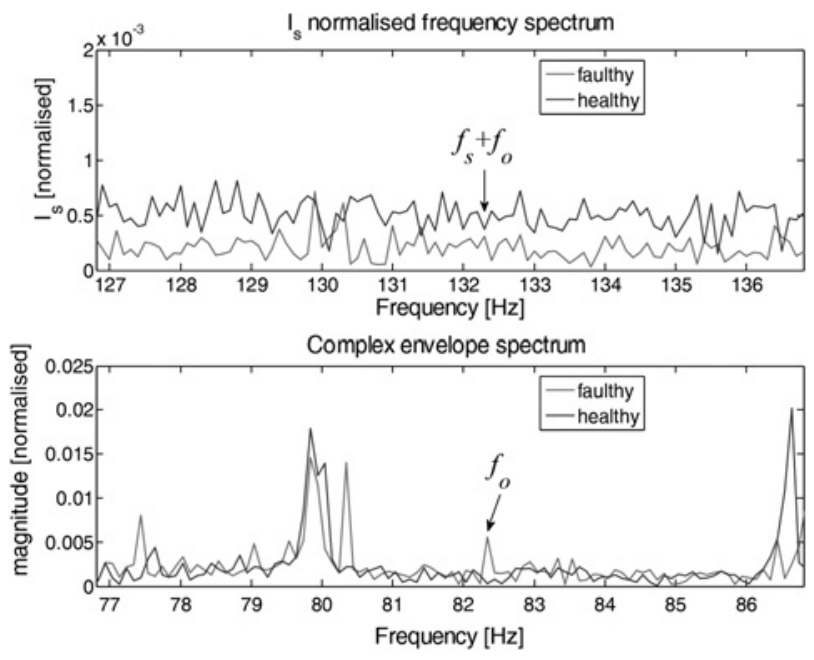

Fig. 11 Stator current (top) and current envelope (bottom) frequency spectrums, $1600 \mathrm{rpm} 12 \mathrm{~mm}$ hole

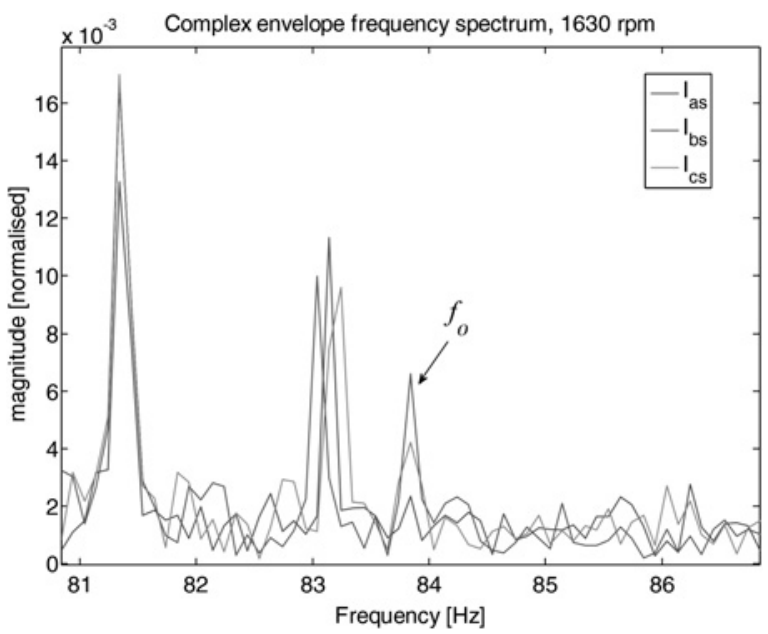

Fig. 12 Stator line currents envelope frequency spectrum, 1630 rpm $12 \mathrm{~mm}$ hole 
not always exhibited at equal magnitudes in all stator line currents. This is illustrated in Fig. 12, where the magnitude of the fault frequency components is seen to vary considerably between the measured machine stator currents. This may make the fault detection difficult if for instance, the signal with the less prominent fault signature were the only available for analysis. The data in Fig. 12 is presented for machine operation at $1630 \mathrm{rpm}$ and a $12 \mathrm{~mm}$ hole located in the bearing outer race. In order to improve the potential for fault detection, the use of the instantaneous negative sequence spectrum was investigated as this method inherently considers the unbalance among the machine phases electrical quantities.

Fig. 13 shows the frequency spectrum of the magnitude of the instantaneous negative sequence obtained using the real valued and complex current signals as inputs in (24). These are compared with the averaged envelope spectrum obtained by separately processing each stator phase complex current signal and then averaging their frequency content. As can be observed in the figure, both negative sequence spectrums have very similar frequency content, with the fault frequency clearly isolated of any adjacent component. The segregation of frequency components is a feature of the symmetrical components. Furthermore, and to the contrary of the complex envelope, the fault frequency in the negative sequence spectra is in fact the larger component in the close vicinity of the fault frequency $f_{\mathrm{o}}$, where the fault component is expected to appear, thus facilitating considerably the fault detection. For an actual bearing fault the isolated localised fault investigated in this work would be accompanied by secondary effects that would further enhance the fault induced air-gap modulation. Hence, it may be expected that the proposed approach could extract a clearer fault signature in real life operating conditions.

The use of instantaneous real valued signals as inputs in the calculation of the symmetrical components does not yield independent values for the negative and positive sequence components. In fact these are the complex conjugate of each other and hence incompatible with the original notion of symmetrical components [19]. For this reason the use of complex signals, in particular analytic complex signals, which yields independent negative and positive components
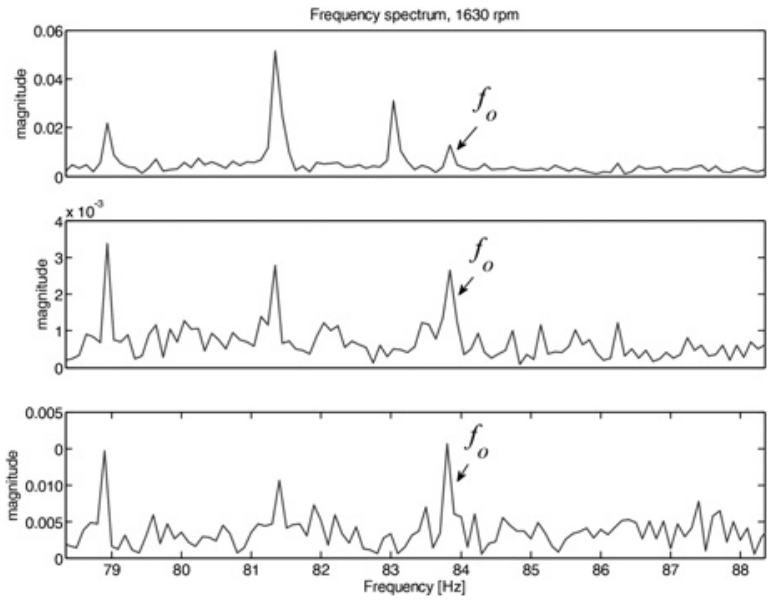

Fig. 13 Complex signals frequency spectrum $1630 \mathrm{rpm} 12 \mathrm{~mm}$ hole

Current envelope average (top), complex signals negative sequence (middle) and real valued signals negative sequence (bottom) has been preferred in the analysis and the simulation of power systems and AC machines [19-21]. However, for purposes of fault detection, both approaches have been found equally effective.

To further assess the effectiveness of use of the instantaneous negative sequence as a fault severity indicator, a series of measurements for bearing faults with different degrees of severity was carried out. Although the artificial fault produced by drilling a hole in the outer race of the bearing gave rise to detectable fault frequencies, the actual air-gap variations induced in the machine were barely noticeable. In order to investigate the effect of a more pronounced air-gap disturbance and avoid physical limitations imposed by the undamaged bearing geometry, three successive grooves spaced by a ball pitch were machined in the bearing outer race, as shown in Fig. 14. This was done in an attempt to emulate the effects of a more profound bearing structure failure. The maximum shaft displacement is controlled by the width of the grooves, and in this way a more direct relationship between air-gap variation and width of the defect, akin to the numerical simulation in Section 3, can be established. The maximum considered groove width was $7 \mathrm{~mm}$, for an estimated temporary static eccentricity of $75 \%$ of the air-gap length in the drive-end of the machine. The upper groove width limit was chosen to minimise the risk of stator-rotor rubbing.

Fig. 15 shows the variation of the amplitude of the fault frequency as a function of the defect width for machine operation at $1630 \mathrm{rpm}$. The presented data demonstrate the

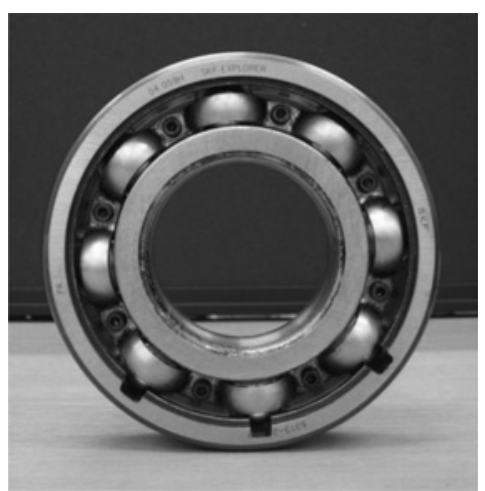

Fig. 14 Artificial bearing fault for fault severity analysis

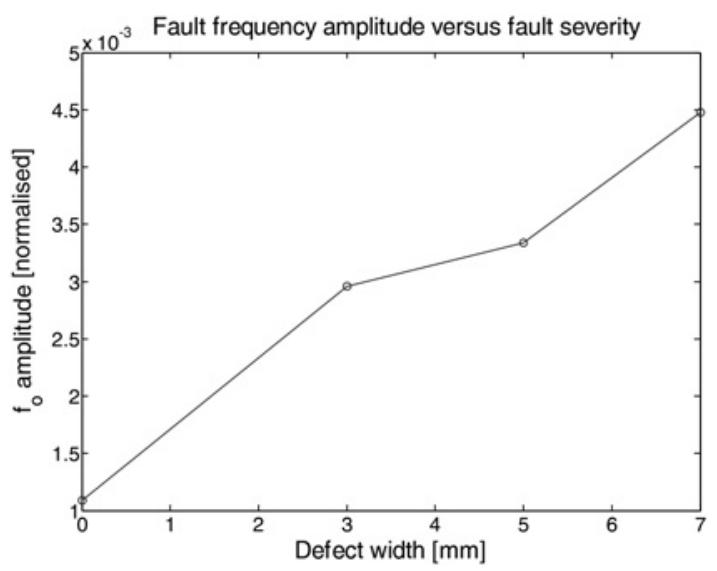

Fig. 15 Fault frequency amplitude against fault severity 
suitability of the use of complex signals as fault severity trend indicators, since the amplitude of the fault frequency increases almost linearly with fault severity. The considered complex signals are of instantaneous nature; hence, any signal analysis technique used in non-stationary signals may be applied to these quantities for enhanced bearing fault detection under variable speed operation.

\section{Conclusions}

This paper investigates the use of electrical signal analysis for the detection of localised outer race bearing faults in a $30 \mathrm{~kW}$ WRIM. A numerical model was developed to facilitate the analysis of incipient bearing fault effects and experimental results were used to verify the findings. The presented analysis demonstrates that conventional CSA is unsuitable for detection of incipient faults and proposes a modified spectral analysis of the complex stator current signal envelope to improve fault detection. The proposed envelope analysis was assessed on the measured faulty machine current signal and shown to offer improvements in the detection of fault specific frequency components for the particular WRIM design investigated in this work. This research also demonstrated that instantaneous negative sequence current signal can provide a further enhancement in bearing fault detection and could have potential for further enhancement using more sophisticated post-processing techniques.

\section{Acknowledgments}

This work was supported by the UK EPSRC as part of the Supergen Wind Consortium under grant EP/H018662/1.

\section{References}

1 Upwind: 'Design limits and solutions for very large wind turbines'. EWEA report, March 2011

2 IEEE Motor reliability working group: 'Report on large motor reliability survey of industrial and commercial installations', IEEE Trans. Ind. Appl., 1985, IA-21, (4), pp. 853-872

3 Alewine, K., Chen, W.: 'Wind turbine generator failure modes analysis and occurrence'. Windpower 2010, Dallas, Texas, 24-26 May 2010

4 Tavner, P.J.: 'Review of condition monitoring of rotating machines', IET Renew. Power Gener., 2008, 2, (4), pp. 215-247

5 Yazidi, A., Henao, H., Capolino, G.A., Betin, F.: 'Rotor inter-turn short circuit fault detection in wound rotor induction machines'. Proc. IEEE ICEM 2010, Rome, Italy, 2010, pp. 1-6

6 Shah, D., Nandi, S., Neti, P.: 'Stator inter-turn fault detection of doubly-fed induction generators using rotor current and search coil voltage signature', IEEE Trans. Ind. Appl., 2009, 45, (5), pp. $1831-1842$

7 Amirat, Y., Choqueuse, V., Benbouzid, M.E.H., Charpentier, J.F. 'Bearing fault detection in DFIG-based wind turbines using the first intrinsic mode function'. Proc. XIX Int. Conf. on Electrical Machines, Rome, Italy, 2010

8 Gong, X., Qiao, W., Zhou, W.: 'Incipient bearing fault detection via wind generator stator current and wavelet filter'. Proc. 36th Annual Conf. IEEE Industrial Electronics Society (IECON 2010), Phoenix, AZ, USA, 7-10 November 2010, pp. 2615-2620

9 Amirat, Y., Choqueuse, V., Benbouzid, M.E.H., Turri, S.: 'Hilbert transform-based bearing failure detection in DFIG-based wind turbines', Int. Rev. Electr. Eng., 2011, 6, (3), pp. 1249-1256

10 Schoen, R.R., Habetler, T.G., Kamran, F., Bartheld, R.G.: 'Motor bearing damage detection using stator current monitoring', IEEE Trans. Ind. Appl., 1995, 31, (6), pp. 1274-1279

11 Blodt, M., Granjon, P., Raison, B., Rostaing, G.: 'Models for bearing damage detection in induction motors using stator current monitoring', IEEE Trans. Ind. Electron., 2008, 55, (4), pp. 1813-1822
12 Gong, X., Qiao, W.: 'Bearing fault detection for direct-drive wind turbines via stator current spectrum analysis'. Energy Conversion Congress and Exposition (ECCE), 2011 IEEE, 17-22 September 2011, pp. 313-318

13 Crabtree, C.J.: 'Survey of commercially available condition monitoring systems for wind turbines' (SuperGen Wind Energy Technologies Consortium, 2010)

14 Williamson, S., Laithwaite, E.R.: 'Generalised harmonic analysis for the steady-state performance of sinusoidally-excited cage induction motors', Proc. IEE, 1985, 132, Part B, (3), pp. 157-163

15 Djurović, S., Williamson, S., Renfrew, A.: 'Dynamic model for doubly-fed induction generators with unbalanced excitation, both with and without faults', IET Electr. Power Appl., 2009, 3, (3), pp. $171-177$

16 Djurović, S., Crabtree, C.J., Tavner, P.J., Smith, A.C.: 'Condition monitoring of wind turbine induction generators with rotor electrical asymmetry', IET Renew. Power Gener., 2012, 6, (4), pp. 207-216

17 Li, X., Wu, Q., Nandi, S.: 'Performance analysis of a three-phase induction machine with inclined static eccentricity', IEEE Trans. Ind. Appl., 2007, 43, (2), pp. 531-541

18 Lyon, W.V.: 'Transient analysis of alternating current machinery' (Wiley, 1954)

19 Yamamura, S.: 'Spiral vector theory of AC circuits and machines' (Clarendon Press, 1992)

20 Stankovic, A.M., Aydin, T.: 'Analysis of asymmetrical faults in power systems using dynamic phasors', IEEE Trans. Power Syst., 2000, 15, (3), pp. 1062-1068

21 Stankovic, V., Sanders, S.R., Aydin, T.: 'Dynamic phasors in modeling and analysis of unbalanced polyphase AC machines', IEEE Trans. Energy Convers., 2002, 17, (1), pp. 107-113

\section{Appendix}

\subsection{Appendix 1}

$$
\begin{aligned}
& i_{\omega_{\mathrm{c}}}(t)=i_{f d} i_{m} \cos \left(\omega_{\mathrm{c}} t+\alpha-\xi\right)+i_{f u} i_{m} \cos \left(\omega_{\mathrm{c}} t-\alpha+\gamma\right) \\
& +i_{f d} i_{m} \cos \left(\omega_{\mathrm{c}} t+\alpha-\xi-\frac{\omega_{\mathrm{c}}}{\omega_{\mathrm{s}}} \frac{\pi}{2}\right) \\
& +i_{f u} i_{m} \cos \left(\omega_{\mathrm{c}} t-\alpha+\gamma-\frac{\omega_{\mathrm{c}}}{\omega_{\mathrm{s}}} \frac{\pi}{2}\right) \\
& i_{\omega_{\mathrm{c}} \pm 2 \omega_{\mathrm{s}}}(t)=i_{f d} i_{m} \cos \left(\left[\omega_{\mathrm{c}}-2 \omega_{\mathrm{s}}\right] t-\alpha-\xi\right) \\
& +i_{f u} i_{m} \cos \left(\left[\omega_{\mathrm{c}}+2 \omega_{\mathrm{s}}\right] t+\alpha+\gamma\right) \\
& +i_{f d} i_{m} \cos \left(\left[\omega_{\mathrm{c}}-2 \omega_{\mathrm{s}}\right] t-\alpha-\xi-\frac{\omega_{\mathrm{c}}}{\omega_{\mathrm{s}}} \frac{\pi}{2}\right) \\
& +i_{f u} i_{m} \cos \left(\left[\omega_{\mathrm{c}}+2 \omega_{\mathrm{s}}\right] t+\alpha+\gamma-\frac{\omega_{\mathrm{c}}}{\omega_{\mathrm{s}}} \frac{\pi}{2}\right) \\
& i_{2 \omega_{\mathrm{c}}}(t)=i_{f d} i_{f u} \cos \left(2 \omega_{\mathrm{c}} t-\xi+\gamma\right) \\
& +i_{f d} i_{f u} \cos \left(2 \omega_{\mathrm{c}} t-\xi+\gamma-\frac{\omega_{\mathrm{c}}}{\omega_{\mathrm{s}}} \frac{\pi}{2}\right) \\
& i_{2\left[\omega_{\mathrm{c}} \pm \omega_{\mathrm{s}}\right]}(t)=\frac{1}{2} i_{f d}^{2} \cos \left(2\left[\omega_{\mathrm{c}}-\omega_{\mathrm{s}}\right] t-2 \xi\right) \\
& +\frac{1}{2} i_{f u}^{2} \cos \left(2\left[\omega_{\mathrm{c}}+\omega_{\mathrm{s}}\right] t+2 \gamma\right) \\
& -\frac{1}{2} i_{f d}^{2} \cos \left(2\left[\omega_{\mathrm{c}}-\omega_{\mathrm{s}}\right] t-2 \xi-2 \frac{\omega_{\mathrm{c}}}{\omega_{\mathrm{s}}} \frac{\pi}{2}\right) \\
& -\frac{1}{2} i_{f u}^{2} \cos \left(2\left[\omega_{\mathrm{c}}+\omega_{\mathrm{s}}\right] t+2 \gamma-2 \frac{\omega_{\mathrm{c}}}{\omega_{\mathrm{s}}} \frac{\pi}{2}\right)
\end{aligned}
$$




\section{www.ietdl.org}

\subsection{Appendix 2}

Test machine parameters

Marelli Motors E4F-225

$240 \mathrm{~V}, 30 \mathrm{~kW}$, 4-poles WRIM

$0.6 \mathrm{~mm}$ air-gap machine winding topology (see Fig. 16).
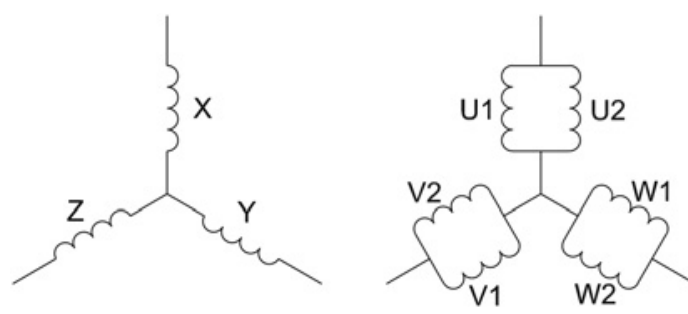

stator

rotor

Fig. 16 Test machine winding topology 